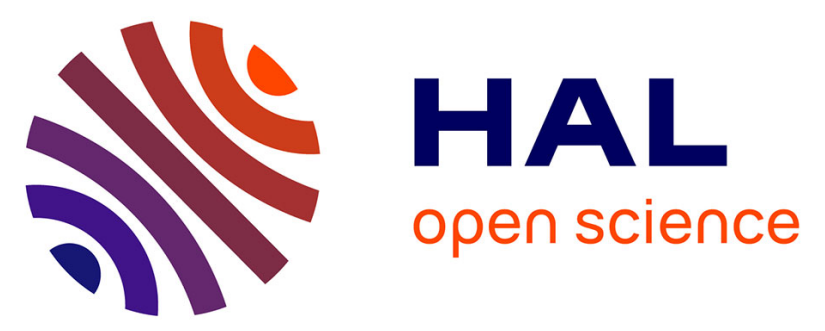

\title{
Effect of functionalization and charging on resonance energy and radial breathing modes of metallic carbon nanotubes
}

S. Öberg, J. Adjizian, D. Erbahar, J. Rio, B. Humbert, M. Dossot, A. Soldatov, S. Lefrant, J.-Y. Mevellec, P. Briddon, et al.

\section{To cite this version:}

S. Öberg, J. Adjizian, D. Erbahar, J. Rio, B. Humbert, et al.. Effect of functionalization and charging on resonance energy and radial breathing modes of metallic carbon nanotubes. Physical Review B: Condensed Matter and Materials Physics (1998-2015), 2016, 93 (4), pp.045408. 10.1103/PhysRevB.93.045408 . hal-01498711

\section{HAL Id: hal-01498711 \\ https://hal.univ-lorraine.fr/hal-01498711}

Submitted on 30 Mar 2017

HAL is a multi-disciplinary open access archive for the deposit and dissemination of scientific research documents, whether they are published or not. The documents may come from teaching and research institutions in France or abroad, or from public or private research centers.
L'archive ouverte pluridisciplinaire HAL, est destinée au dépôt et à la diffusion de documents scientifiques de niveau recherche, publiés ou non, émanant des établissements d'enseignement et de recherche français ou étrangers, des laboratoires publics ou privés. 


\title{
Effect of functionalization and charging on resonance energy and radial breathing modes of metallic carbon nanotubes
}

\author{
S. Öberg, ${ }^{1}$ J.-J. Adjizian, ${ }^{2,3}$ D. Erbahar,,${ }^{3,4}$ J. Rio, ${ }^{3}$ B. Humbert, ${ }^{3}$ M. Dossot,${ }^{5}$ A. Soldatov, ${ }^{1,6}$ S. Lefrant,,${ }^{3}$ J.-Y. Mevellec, ${ }^{3}$ \\ P. Briddon, ${ }^{3,7}$ M. J. Rayson, ${ }^{7}$ and C. P. Ewels ${ }^{3, *}$ \\ ${ }^{1}$ Department of Engineering Sciences \& Mathematics, Luleå University of Technology, SE - 97187, Luleå, Sweden \\ ${ }^{2}$ Nanoscopic Physics (NAPS), Université catholique de Louvain, Place de l'Université 1 B-1348 Louvain-la-Neuve, Belgique \\ ${ }^{3}$ Institut des Matériaux Jean Rouxel (IMN), Université de Nantes, Centre national de la recherche scientifique (CNRS), \\ 2 rue de la Houssinière, BP32229, 44322 Nantes, France \\ ${ }^{4}$ Physics Department, Gebze Technical University, 41400 Kocaeli, Turkey \\ ${ }^{5}$ Laboratoire de Chimie Physique et Microbiologie pour l'Environnement (LCPME), UMR 7564 CNRS-Université de Lorraine, \\ 405 rue de Vandoeuvre, 54600 Villers-les-Nancy, France \\ ${ }^{6}$ Department of Physics, Harvard University, Cambridge, Massachusetts 02138, USA \\ ${ }^{7}$ School of Electrical and Electronic Engineering, University of Newcastle, Newcastle upon Tyne, NE 1 7RU, United Kingdom
}

(Received 8 October 2015; published 11 January 2016)

\begin{abstract}
While changes in resonant Raman scattering measurements are commonly used to measure the effect of chemical functionalization on single-walled carbon nanotubes, the precise effects of functionalization on these spectra have yet to be clearly identified. In this density functional theory study, we explore the effects of functionalization on both the nanotube resonance energy and frequency shifts in radial breathing mode. Charge transfer effects cause a shift in the first Van Hove singularity spacings, and hence resonance excitation energy, and lead to a decrease in the radial breathing mode frequency, notably when the Fermi level decreases. By varying stochastically the effective mass of carbon atoms in the tube, we simulate the mass effect of functionalization on breathing mode frequency. Finally, full density functional calculations are performed for different nanotubes with varying functional group distribution and concentration using fluorination and hydrogenation, allowing us to determine overall effect on radial breathing mode and charge transfer. The results concur well with experiment, and we discuss the importance when using Raman spectroscopy to interpret experimental functionalization treatments.
\end{abstract}

DOI: 10.1103/PhysRevB.93.045408

\section{INTRODUCTION}

Single-walled carbon nanotubes (SWCNTs) have tremendous mechanical and electronic properties with potential application in many different areas [1-3]. It is often desirable to tune the nanotube properties, which is typically achieved through chemical functionalization [4-6]. However, it remains difficult to accurately determine the nature and degree of chemical functionalization.

Resonant Raman spectroscopy is commonly used as proof of chemical functionalization of SWCNTs [7]. The SWCNTs show three key single-phonon absorption bands [8]. The G band, at $\sim 1590 \mathrm{~cm}^{-1}$, is associated with collective tangential $\mathrm{C}=\mathrm{C}$ bond vibrations in benzenoid rings and is always present in aromatic $\mathrm{sp}^{2}$ carbon systems such as nanotubes. The D band at $1250-1350 \mathrm{~cm}^{-1}$ is associated with defects and damage, causing localization and symmetry breaking [9]. This band is forbidden in first-order Raman spectra, and it can only be seen in a second-order Raman process [10]. Finally, nanotubes exhibit a low frequency $\left(100-400 \mathrm{~cm}^{-1}\right)$ radial breathing mode (RBM) associated with a radial expansion and contraction of the nanotube.

The RBM frequency is inversely proportional to the tube diameter as follows:

$$
v\left(\mathrm{~cm}^{-1}\right)=\frac{A\left(\mathrm{~cm}^{-1} \cdot \mathrm{nm}\right)}{d}+B\left(\mathrm{~cm}^{-1}\right) .
$$

\footnotetext{
*Corresponding author: chris.ewels@ cnrs-imn.fr
}

The precise values for parameters $A$ and $B$ vary, notably $B$ depends on environmental interactions such as tube bundling, solvents, and substrate. The first theoretically predicted values for $A$ and $B$ were 234 and $0 \mathrm{~cm}^{-1}$.nm, respectively [11]. Other typically quoted values are $A=224 \mathrm{~cm}^{-1}$. nm, $B=14 \mathrm{~cm}^{-1}$ [12] and $A=234 \mathrm{~cm}^{-1}$.nm, $B=10 \mathrm{~cm}^{-1}$ for SWCNT bundles in the diameter range; $d=1.5 \pm 0.2 \mathrm{~nm}$ [14] (where $B$ is an upshift coming from tube-tube interactions), $A=$ $248 \mathrm{~cm}^{-1}$.nm and $B=0 \mathrm{~cm}^{-1}$ for tubes with diameters above $0.7 \mathrm{~nm}$ [15], and $A=223.5, B=12.5$ for isolated SWCNTs on oxidized $\mathrm{Si}$ substrates [13]. An alternative RBM fit accounting for environmental effects has been proposed of the form [15],

$$
v\left(\mathrm{~cm}^{-1}\right)=\frac{227 \mathrm{~cm}^{-1} \cdot \mathrm{nm}}{d} \sqrt{1+C d^{2}} .
$$

Due to their 1D nature, SWCNTs have Van Hove singularities in their electronic density of states. When the Raman excitation laser energy matches the spacing between such peaks (typically the $M_{11}$ transition for metallic tubes and $S_{22}$ or $S_{33}$ for semiconducting tubes), the Raman scattering process becomes resonant, and signal intensity typically increases by a factor of $10^{6}$. Typically only nanotubes in resonance with the laser are observed. The spacing of Van Hove singularities depends on the nanotube chirality, and hence it is possible to predict which chirality nanotubes will be in resonance for a given laser excitation energy [16].

The absence of the RBM is often used as an indication of the chemical response of a nanotube to a particular 
functionalization treatment, i.e., if the RBM is present before chemical treatment and is absent afterwards, this is commonly taken as a sign that the tube has become significantly chemically functionalized so that the RBM is no longer active. This can be due to the formation of many $\mathrm{sp}^{3}$ defects and consequent changes in the electronic and phonon density of states and also shifts in the resonance window due to charge transfer.

With the use of a tunable laser, it is possible to determine how much the resonance energy for a given tube has changed with functionalization, along with the shift in vibrational frequency of the tube [8]. Previous work has shown that as tube functionalization increases, RBM intensities decrease while their frequencies increase $[15,17]$. The change in resonance is due to the functionalization itself but also to the presence of electron acceptor in the chain near the tube acting as a dopant. Azomethine ylide functionalization was shown to upshift the RBM of semiconducting tubes by a few wavenumbers with negligible effect on metallic tubes [18]; however, in contrast, our recent study showed a $3-4 \mathrm{~cm}^{-1} \mathrm{RBM}$ upshift upon reductive alkylation and $7-8 \mathrm{~cm}^{-1}$ for esterification with a stronger effect in metallic tubes [17]. The RBM upshifts of $\sim 8 \mathrm{~cm}^{-1}$ were observed for $-\mathrm{COOH}$ functionalized SWCNTs, ascribed to increased stacking interaction in SWCNT bundles [19], with similar $2-7 \mathrm{~cm}^{-1}$ upshifts in small-diameter $(0.7 \mathrm{~nm})$ SWCNTs after $5 \%$ functionalization with aryl diazonium salts [20]. Similarly, nanotube water filling (both intentional and accidental) has also been shown to upshift RBM values [21], and it is likely that changes in the aggregation state of a given nanotube with chemical functionalization might also influence the RBM frequency.

In contrast, low dose fluorination downshifts RBMs [22,23] and calculations for axial lines of epoxide oxygen along a $(10,0)$ showed a drop in the RBM of $16-18 \mathrm{~cm}^{-1}$ [24]. Finally, pentyl and tert-butyl functionalized tubes showed no observable shift in RBM [25].

Chemical functionalization can potentially induce a number of different effects in the nanotube (including changing the effective mass of the carbon atoms in the wall, distorting the tube cross section, charge transfer effects, etc.), and it is difficult to experimentally extract each of these effects and determine their relative importance. In this paper, via density functional computer modelling, we examine in detail charge transfer effects on the spacing of the first Van Hove singularities and hence the resonance excitation energy, the mass effect of functionalization with both hydrogen and fluorine on breathing mode frequency, and finally, by varying functional group distribution, we determine overall effect on RBM and charge transfer. These results are of primary importance to be able to understand evolution of Raman spectra for functionalized SWCNTs in relation to the chemical nature of the covalently attached groups and their distribution.

\section{METHOD}

We use spin polarized density functional theory (DFT) calculations under the local density approximation (LDA) [26], as implemented in the AIMPRO package [27-30]. The 24 carbon atom calculations (Secs. III A and IIIB) were carried out using large hexagonal supercells ( $a=40$ a.u., $c=\sim 4.6$ a.u. depending on optimization), fitting the charge density to plane waves within an energy cutoff of $300 \mathrm{Ha}$. Charge density oscillations in partly filled degenerate orbitals during the self-consistency cycle were damped using a MethfesselPaxton smearing function with $k_{B} T=0.005 \mathrm{eV}$. Relativistic pseudopotentials are taken from the Hartwingster-GoedeckerHutter scheme [31]. Atom-centered Gaussian basis functions are used to construct the many-electron wave function. These functions are labeled by multiple orbital symbols, where each symbol represents a Gaussian function multiplied by polynomial functions, including all angular momenta up to maxima $p(l=0,1), d(l=0,1,2)$, and $f(l=0,1,2,3)$, respectively. Following this nomenclature, the basis sets used for each atom type were $p d d d p(\mathrm{C}), p p p(\mathrm{H})$, and $d d p p(\mathrm{~F})$, with increasing functional localization from left to right (a more detailed discussion of the basis functions can be found in Ref. [32]). A Bloch sum of these functions is performed over the lattice vectors to satisfy the periodic boundary conditions of the supercell. Atom positions and axial unit cell length are simultaneously optimized with no symmetry constraints. Density of states is plotted with $0.07 \mathrm{eV}$ Gaussian broadening.

Calculations such as these exploring frequency differences of only a few wavenumbers require an extremely high level of precision, and it is for this reason that we have chosen such dense k-point grids (a $1 \times 1 \times 60$ grid with a $1 \times 1 \times 240$ extrapolated grid used for density of states calculations), high plane wave energy cutoff (300 $\mathrm{Ha})$, and high convergence tolerances for self-consistency $\left(10^{-8} \mathrm{Ha}\right)$, energy $\left(10^{-8} \mathrm{Ha}\right)$, and position convergence $\left(10^{-7}\right.$ a.u.). Additional precision is gained since our primary interest is in relative frequencies as we change properties such as mass or charge state, in which case some absolute errors, for example, through choice of exchange-correlation functional, cancel out. Nonetheless, we note that where direct comparison with experiment is possible (e.g., the ${ }^{13} \mathrm{C}$ results in Sec. III B), our absolute values match to within $2 \mathrm{~cm}^{-1}$. The large lateral cell size minimizes the effect of the uniform background charge necessarily applied during Fermi level shift calculations.

Vibrational frequencies and normal modes are obtained as follows. Each atom in a relaxed molecular structure is displaced in the $x, y$, and $z$ directions by 0.2 a.u. in turn, and the forces on all atoms are evaluated analytically. The second derivative of the energy with respect to the positions of atoms $i$ and $j, d^{2} E / d R_{i} d R_{j}$, is then obtained by a finite difference formulation of the derivative using the calculated forces. From these numerical second derivatives, the dynamical matrix can be built up by dividing each term by the product of the masses of atoms $i$ and $j,\left(d^{2} E / d R_{i} d R_{j}\right) / M_{i} M_{j}$, and the subsequent eigenvalue problem is solved in the usual way.

The large cell calculations discussed in Sec. III C contain $\mathrm{C}_{144} \mathrm{H}_{n}, n=0-14$ atoms in the supercell, and their size necessitates lower levels of precision to remain computationally accessible. The hexagonal cell dimensions were $a=19.050 \AA, c=14.965 \AA$ along the tube axis, and gamma point calculations with $0.01 \mathrm{eV}$ finite temperature Fermi occupation function, with energy tolerance cutoffs of $10^{-7} \mathrm{Ha}$ for self-consistency cycle and energy convergence during optimization. 

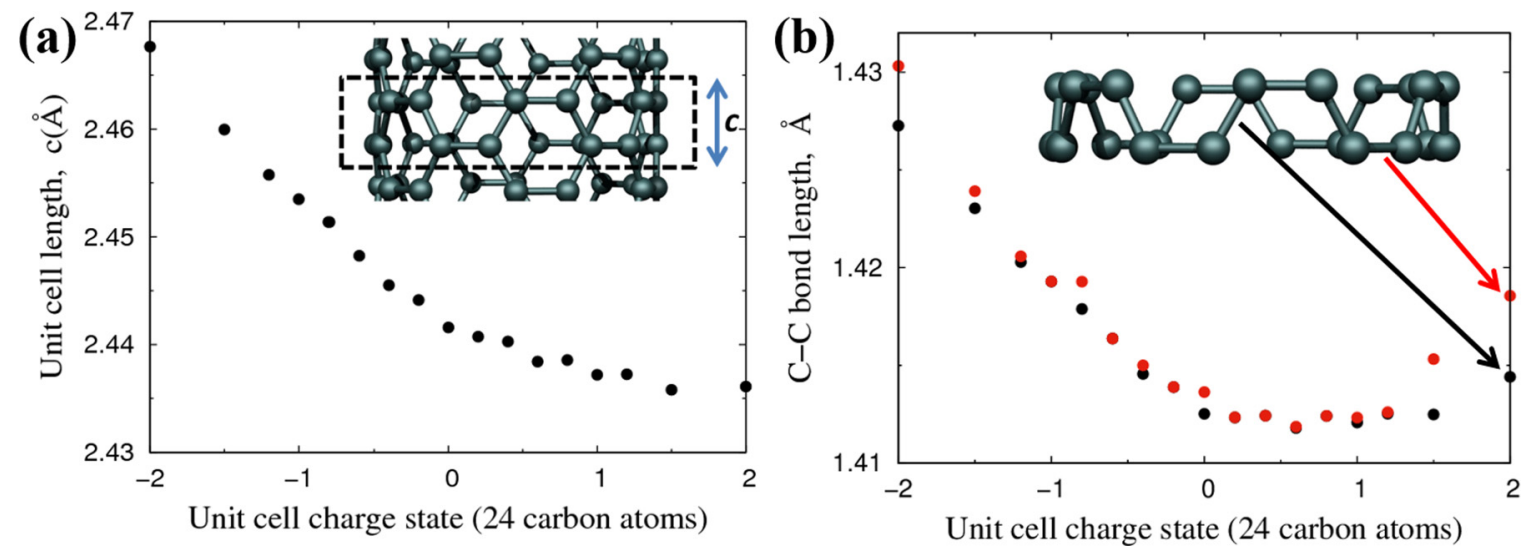

FIG. 1. Variation in (a) geometrically optimized unit cell length, $c(\AA)$, and (b) C- C bond length $(\AA)$ for a $(6,6)$ carbon nanotube as a function of Fermi level shift. Red (black) points in (b) indicate bonds that are parallel (at an angle) to the nanotube axis.

\section{RESULTS}

\section{A. Effect of charging}

We first explore the effect of charge transfer from dopants to the nanotube. A generalized Fermi level shift is expected to be a more relevant model for doping through chemical functionalization in metallic than semiconducting tubes due to the enhanced charge delocalisation in metallic tubes [33]. We used a pristine $(6,6)$ metallic nanotube containing 24 atoms in the tube unit cell. We then shifted the Fermi level, reoptimising atom positions and unit cell length each time. As expected, our LDA bond lengths are slightly shorter than generalized gradient approximation (GGA) values from the literature of $1.423 / 1.427 \AA$ [34]. The nanotube shows weak piezoelectric behavior, expanding and contracting with charging [(Fig. 1(a)], which is consistent with trends seen in previous $\pm 0.01 \mathrm{e} / \mathrm{C}$ low charge injection calculations [35]. Notably, the behavior is linear and more pronounced for negative charging. This behavior is reflected in the $\mathrm{C}-\mathrm{C}$ bond lengths [Fig. 1(b)], which also increase linearly with negative charging, but shows little variation for positive charging until gradually extending for large downwards Fermi level shifts.

This geometry variation is reflected in the calculated RBM of the nanotube (Fig. 2), which decreases approximately linearly with negative charging, stays approximately constant with weak positive charging, and then rapidly drops at higher positive charging. Nonetheless, the magnitude of these changes is small, and charging the nanotube does not cause significant RBM variation except at large positive charging. The best fit we obtain over the linear region (excluding the lower RBM outrider points) is $v=1.885 x+283.01 \mathrm{~cm}^{-1}$, where $x$ is the charge per unit cell ( 24 carbon atoms). This serves as a measure of the RBM change through charge transfer doping effects from functionalization or the surrounding environment. The large asymmetric calculated downshift in RBM upon electron donation by the tube is interesting. It matches well with experimental observations in iodine filled SWCNTs, where a downshift of $7 \mathrm{~cm}^{-1}$ of the RBM from $158 \mathrm{~cm}^{-1}$ to $151 \mathrm{~cm}^{-1}$ was seen upon iodine filling of the tubes [36]. This frequency, excited via a $785 \mathrm{~nm}$ laser excitation, corresponds to a $M_{11}$ transition for a $(19,1)$ metallic nanotube.

The electronic density of states of the tube is shown in Fig. 3, where the first Van Hove spacing, $M_{11}$, is marked. The energy scale is shifted for clarity, aligning the center of $M_{11}$ at zero in each case, and the Fermi level is marked with a dot. The very low density of states around the Fermi level means the Fermi energy shifts rapidly with charging. When charging reaches 0.8 e per unit cell or above (either positive or negative), the Fermi level moves into the first Van Hove singularity peak. This will lead to attenuating of the resonant behavior as states are no longer available for $M_{11}$ transitions, consistent with a loss of RBM intensity such as that seen experimentally with functionalization [17]. However, the overall form of the Van Hove peaks remains the same, with no appreciable increase in full width at half maximum (FWHM), suggesting chargetransfer effects will not induce smearing-related intensity loss in resonant Raman intensity.

The Raman resonance profile is governed by the equation,

$$
I\left(E_{L}\right) \alpha \frac{C_{ \pm}\left(E_{L}, \hbar \omega\right)}{\left|\left(E_{L}-E_{i i}-i \Gamma\right)\left(E_{L} \pm \hbar \omega-E_{i i}-i \Gamma\right)\right|^{2}},
$$

where $E_{L}$ is the laser energy, $E_{i i}$ is the energy of the optical transition, $\hbar \omega$ is the phonon energy, $\Gamma$ is proportional to the lifetime of the intermediate electronic states, and $C_{ \pm}$is a scale factor [37]. $\Gamma$ increases upon nanotube functionalization,

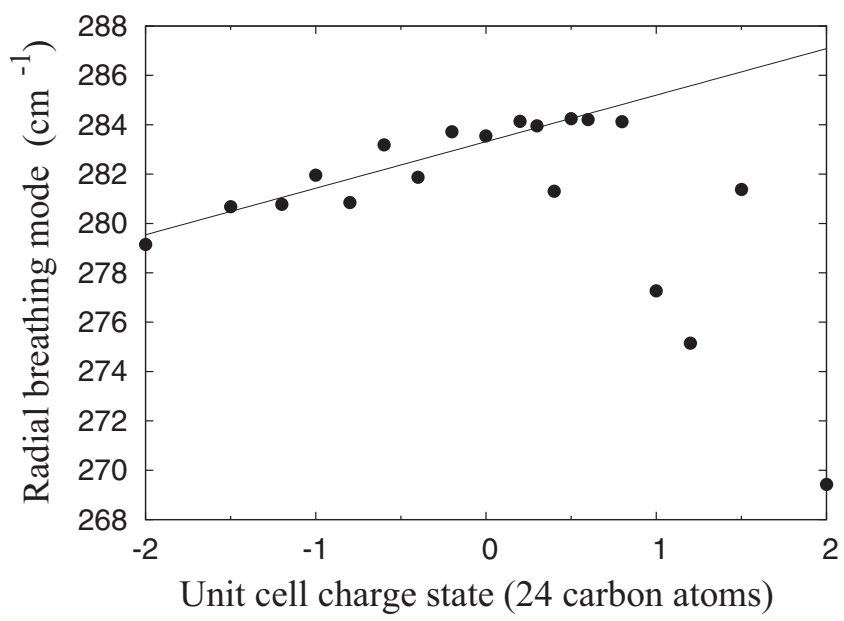

FIG. 2. Variation in RBM $\left(\mathrm{cm}^{-1}\right)$ for a $(6,6)$ metallic carbon nanotube, as a function of unit cell charge state. 


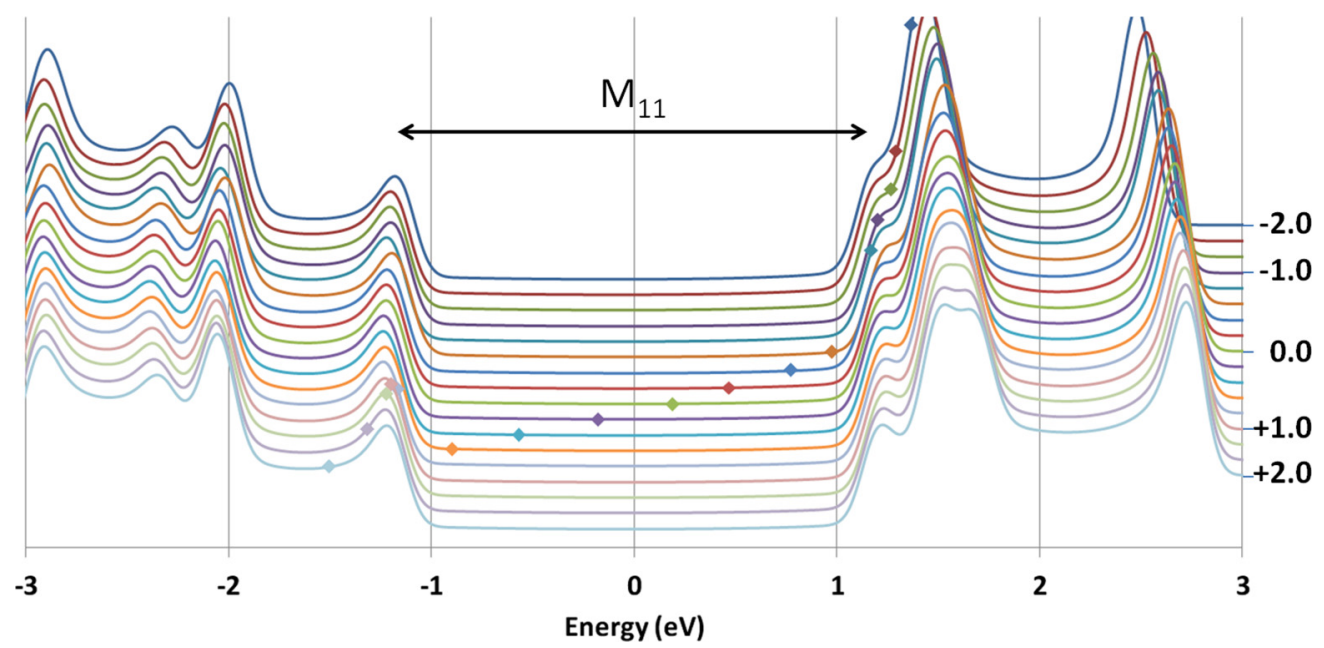

FIG. 3. Density of states for $(6,6)$ metallic nanotube as a function of unit cell charge state ( 24 carbon atoms), as marked on the right-hand axis. Densities are aligned so that the midpoint of the $M_{11}$ first Van Hove transition is at $0 \mathrm{eV}$. The Fermi level is marked in each case with a diamond. Charge states are $-2.0,-1.5,-1.2,-1.0,-0.8,-0.6,-0.4,-0.2,0,+0.2,+0.4,+0.6,+0.8,+1.0,+1.2,+1.5$, and +2.0 .

resulting in a decrease in signal intensity. These calculations show, however, that this change in $\Gamma$ is not due to a general shift in the Fermi level during functionalization since the FWHM for the first Van Hove singularities does not change. Instead, it must be a localized effect associated with the bonding during functionalization.

Figure 4 shows there is a general downward trend in the $M_{11}$ Van Hove spacing, with both positive and negative charging, up to $86 \mathrm{meV}$ for -2 charge per $24 \mathrm{C}$ atoms and $20 \mathrm{meV}$ for +2 charge. Thus, simple Fermi level shifting predicts a redshift in the resonance excitation energy with both donor and acceptor doping for metallic nanotubes. This is consistent with our previous experimental findings that show a decrease in resonance energy of 21 and $43 \mathrm{meV}$ for reductive alkylation and esterification, respectively [17].

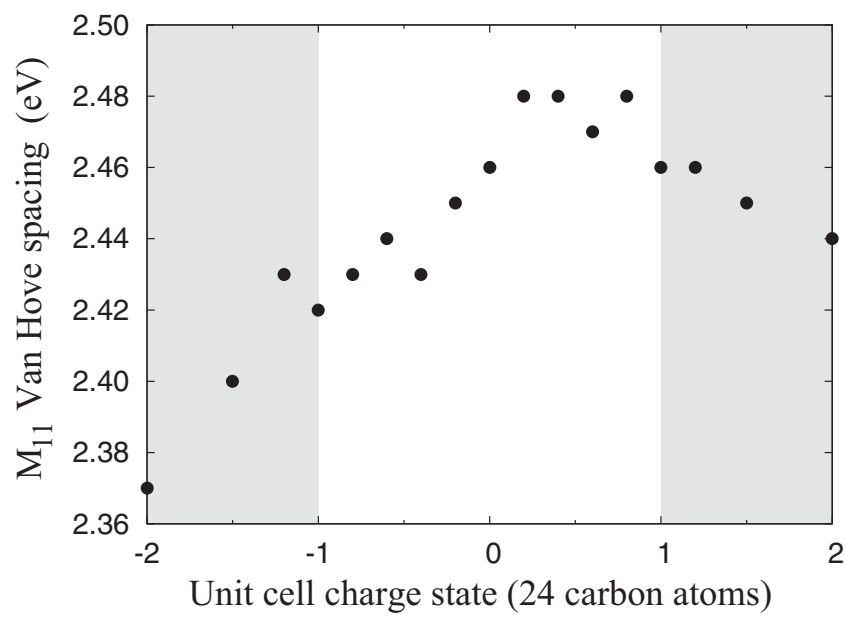

FIG. 4. Calculated variation in $M_{11}$ first Van Hove spacing $(\mathrm{eV})$ from the density of states in Fig. 3 for $(6,6)$ carbon nanotube, as a function of unit cell charge state (per 24C atoms). At charging levels beyond \pm 1 electron per 24 carbon atoms (shaded zones) this transition is no longer allowed since the Fermi level has swept through the first Van Hove peak.
To conclude the examination of the effects on RBM behavior of charge transfer and corresponding Fermi level shift, it appears that this can semiquantitatively account for the experimentally observed redshifts in resonance energy upon functionalization, along with the observed signal intensity decrease. However, the experimentally observed frequency shifts cannot be satisfactorily explained simply through charge transfer, so we next examine the effect of mass variation caused by chemical functionalization.

\section{B. Effect of mass variation in the tube}

We next turn our attention to the effect of mass variation on the RBM. As discussed in the Sec. II, the dynamical matrix is constructed by dividing each term in the second derivative of the energy with respect to the positions of atoms $i$ and $j$ by the product of the masses of atoms $i$ and $j,\left(d^{2} E / d R_{i} d R_{j}\right) / M_{i} M_{j}$. The eigenvalues of this matrix give the vibrational modes of the nanotube. For the previous section, we used carbon atom masses $M_{i}=M_{j}=12$. In the current section, we calculate the second derivative of the energy for a pristine 24 atom unit cell of a $(6,6)$ carbon nanotube and then examine the effect on the calculated RBM of varying the masses $M$. A chosen percentage of carbon atoms are allocated an alternative mass and the RBM calculated. The specific carbon atoms are chosen randomly; this procedure is repeated 200 times in order to explore the importance of specific site selection on the resultant RBM.

In a first approximation, we assume that the effect of functionalizing a carbon atom in the tube is simply to increase the mass of that atom, i.e., assuming that the atom and its functional group are vibrating as a rigid unit. Figure 5 shows the result of randomly replacing ${ }^{12} \mathrm{C}$ atoms in the tube with atoms that are $10 \%$ heavier. This shows that, as might be expected, the RBM drops as the fraction of heavy sites increases. A best fit to these values gives the function $v=-0.137 x+283.5 \mathrm{~cm}^{-1}$, where $x$ is the percentage of functionalized sites. Thus, the rigid addition model for mass considered here suggests that 


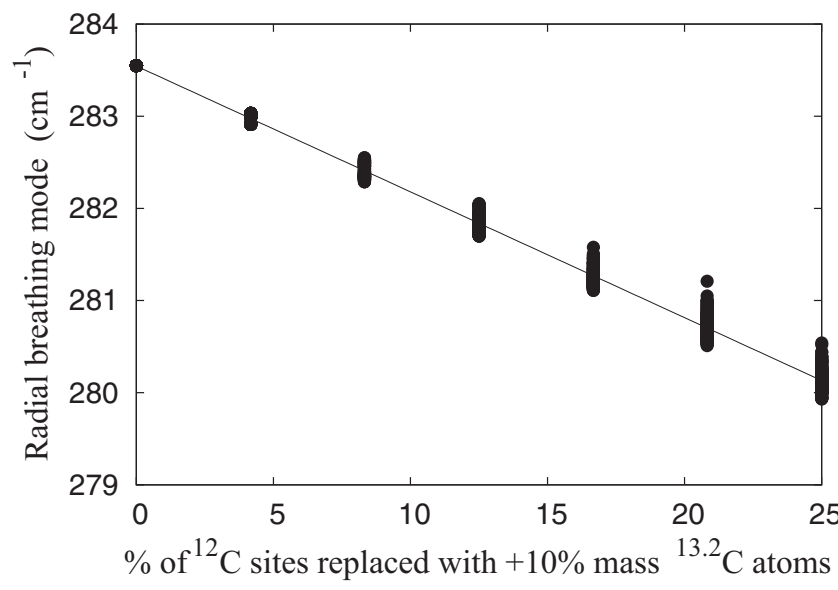

FIG. 5. Variation in calculated RBM for $(6,6)$ carbon nanotube as a function of mass of carbon atoms in the unit cell. Default atom mass is $12 ; n$ sites are randomly selected $(n=0-6)$ and assigned an atom mass of $13.2(+10 \%)$. Multiple points for each value of $n$ reflect different random site selection.

the RBM could drop by several wavenumbers if, for example, the tube was heavily hydrogenated.

This approach also allows us to explore the effect of the site distribution of functional groups, as represented by the spread in calculated RBMs for a given percentage of increased mass. The results show that even for extremely high functionalization fractions of $25 \%$, the spread in calculated RBM as a function of site distribution is less than $1 \mathrm{~cm}^{-1}$. Thus, we conclude that in terms of their mass, the precise spatial distribution of functional sites should not significantly affect the RBM of the functionalized tube. The effect of site distribution when the tube is allowed to geometrically optimize after functionalization is not included here and is explored below.

Incidentally, these results confirm the negligible effect of natural abundance of ${ }^{13} \mathrm{C}$ on the $\mathrm{RBM}$, which will cause a decrease in the RBM of less than $0.2 \mathrm{~cm}^{-1}$ and a broadening of less than $1 \mathrm{~cm}^{-1}$. Our calculated RBM for a fully ${ }^{13} \mathrm{C}(6,6)$ nanotube is $272.4 \mathrm{~cm}^{-1}$, a decrease of just over $10 \mathrm{~cm}^{-1}$ compared to the ${ }^{12} \mathrm{C}$ value of $283.6 \mathrm{~cm}^{-1}$. A recent experimental study of ${ }^{13} \mathrm{C}$ isotope enriched nanotubes found a relation of $v=A / d \mathrm{~cm}^{-1}$, where $A=232 \mathrm{~nm} \cdot \mathrm{cm}^{-1}$ for fully ${ }^{12} \mathrm{C}$ nanotubes and $222.6 \mathrm{~nm} \cdot \mathrm{cm}^{-1}$ for fully ${ }^{13} \mathrm{C}$ nanotubes [38]. This gives experimental RBM frequencies for a $(6,6)$ tube of 285 and $273 \mathrm{~cm}^{-1}$, respectively, in extremely close agreement with our calculated values.

This analysis of rigid unit functionalization is somewhat simplistic. An alternative situation is that bulky, high mass functional groups may be added to the nanotube surface, which do not vibrate in synchronization with the carbon atoms but instead serve as a block to radial motion of the carbon atom. In this case, the net effect would be to increase vibrational frequency and effectively reduce the carbon net mass. A similar situation has been discussed for double walled nanotubes where the internal and external tubes are considered as coupled oscillators, with the external tube constraining the internal tube and increasing its RBM frequency [39].

Indeed, when we repeat the above calculations, with randomized masses reduced $10 \%$ to ${ }^{10.8} \mathrm{C}$, we indeed see

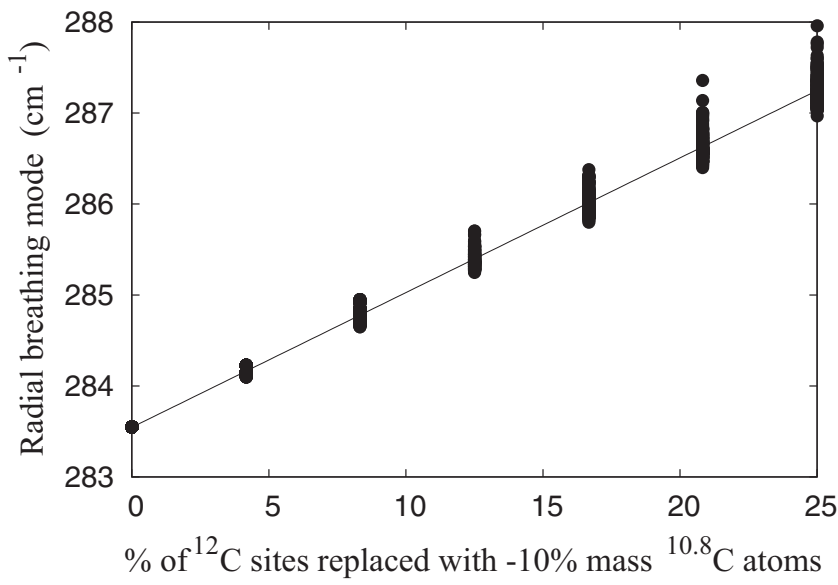

FIG. 6. Variation in calculated RBM for $(6,6)$ carbon nanotube, as a function of mass of carbon atoms in the unit cell. Default atom mass is $12 ; n$ sites are randomly selected $(n=0-6)$ and assigned an atom mass of $10.8(-10 \%)$. Multiple points for each value of $n$ reflect different random site selection.

the RBM frequency increasing, with a best-fit form of $v=+0.148 x+283.6 \mathrm{~cm}^{-1}$, where $x$ is the percentage of functionalized sites (Fig. 6).

These two situations were observed in a comparative study of the $\mathrm{C}-\mathrm{H}$ stretch mode in $\mathrm{HCN}$ and an $\mathrm{H}$ passivated substitutional $\mathrm{C}$ impurity in GaAs [40]. In this case, the stretch frequency $v$ of the $\mathrm{C}-\mathrm{H}$ bond is given by $v^{2}=$ $k\left(1 / M_{H}+1 / \chi M_{C}\right)$, where $k$ is the force constant of the oscillator and $\chi$ is the carbon effective mass parameter, dependent on the backbonding of the carbon atom. In HCN, $\chi$ was found to be less than 1 (0.77) due to the exceptionally strong $\mathrm{C} \equiv \mathrm{N}$ bond, whereas for substitutional carbon in GaAs, where the back $\mathrm{Ga}-\mathrm{C}$ bonds are soft and angled to the $\mathrm{CH}$ bond, $\chi$ is greater than $1(1.17-1.23)$. It was therefore suggested that $\chi$ values below one were indicative of strong backbonding of the carbon.

In summary, these results suggest that the effective masses of the functionalized carbon atoms in the nanotube, and their resultant effect on the nanotube RBM frequency, should depend on the nature of the functionalization. If the functional group is considered to vibrate synchronously with the carbon atom as a rigid unit, then the RBM drops, in our example by several wavenumbers even when the mass change is relatively small if the functionalization fraction is relatively high. Alternatively, if the functional group is considered as a larger obstacle blocking the radial carbon motion, and decreasing the effective mass of the carbon, then the inverse effect is seen, and the RBM increases.

To understand and distinguish between these models, it is necessary to move to the next logical step, i.e., to calculate the $\mathrm{RBM}$ of functionalized nanotubes.

\section{Full calculations for hydrogenated and fluorinated nanotubes}

In this final section, we perform full calculations of hydrogenated and fluorinated $(6,6)$ nanotubes in order to fully incorporate all effects (charge transfer, changes in local 

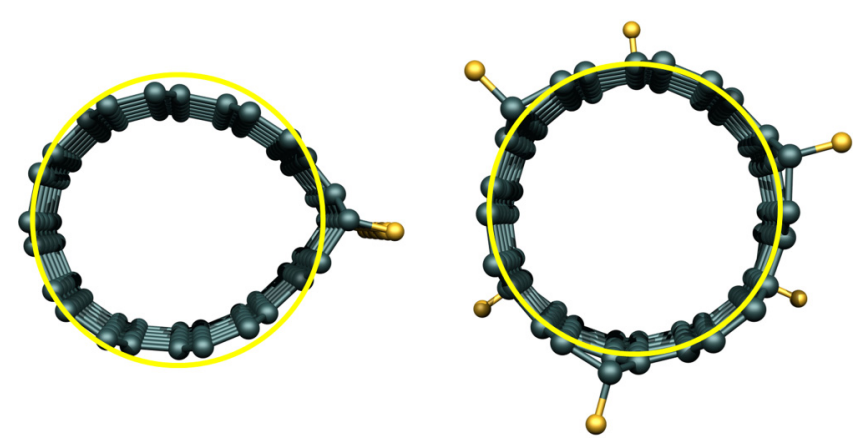

FIG. 7. Change in cross section of $(6,6)$ carbon nanotube with one carbon site fluorinated in every 24 (left) using the 24 atom unit cell, resulting in a line of fluorinated sites along the tube axis, and (right) using six repeated unit cells, 144 carbon atoms, of which six random sites fluorinated. Yellow circle indicates fully circular cross section for reference.

bonding, and changes in effective mass). For such calculations, it is no longer possible to work with a single unit cell since the repeat vector along the nanotube axis is too short. Thus, every functional group addition effectively adds a functionalized line along the tube axis, which results in unphysical distortion to the nanotube cross section. For this reason, we have used larger 144 atom supercells for this section, despite their significantly increased computational cost (see Fig. 7).

The calculated RBM frequency as a function of degree of nanotube functionalization is shown in Fig. 8. The increased noise in the values than previous sections is because of the lower cutoffs and tolerances applied, an unfortunate computational necessity due to the size of the calculations. The sites to functionalize are chosen randomly with the constraint that neighboring sites cannot be selected. This approach is supported by Sec. III B results, which suggest that the exact site distribution should not significantly alter the calculated RBM, although it may also be a contributing factor to the observed noise.

The results show that there is a general linear downward trend in RBM with increasing hydrogenation, the best fit giving $v=-0.948 x+283.4 \mathrm{~cm}^{-1}$, where $x$ is the percentage of

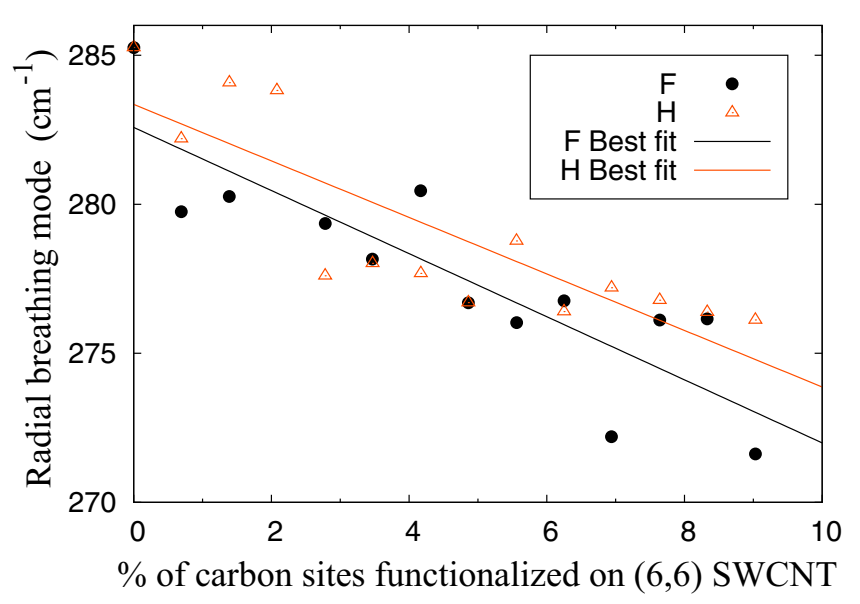

FIG. 8. Calculated RBM for a $(6,6)$ carbon nanotube as a function of varying degree of hydrogenation and fluorination.

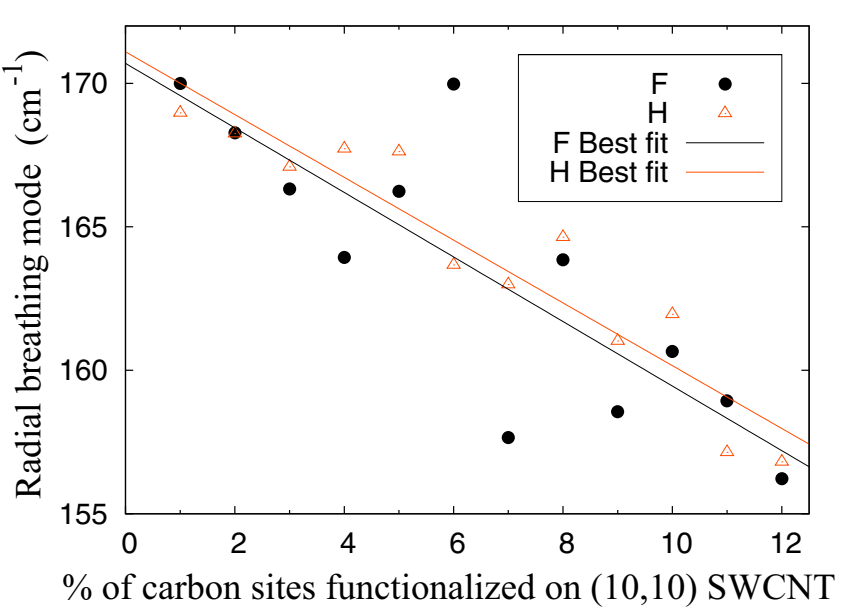

FIG. 9. Calculated RBM for a $(10,10)$ carbon nanotube as a function of varying degree of hydrogenation and fluorination.

hydrogenated sites. Comparing this with the variable mass calculations in Sec. III B suggests that the effective mass change of the $\mathrm{C}$ atoms is only responsible for $\sim 15 \%$ of the calculated change in RBM, with the rest therefore being due to charge transfer and structural modification to the carbon nanotube (pyramidalization and weakening of $\mathrm{C}-\mathrm{C}$ bonding). We note that the eigenvectors associated with these RBMs are not as symmetrically clean as that of the pristine tube, and in each case we have identified the most symmetric radial mode as the RBM. There is a surprising degree of similarity in RBM frequency behavior with fluorination, again showing a linear decrease with degree of fluorination, the best fit giving $v=-1.059 x+282.6 \mathrm{~cm}^{-1}$, where $x$ is the percentage of fluorinated sites. This is in good agreement with Ref. [23], which shows an experimentally observed RBM downshift upon fluorination of $12 \mathrm{~cm}^{-1}$. Thus, for these atomic functional groups, it seems that each $1 \mathrm{~cm}^{-1}$ downshift in RBM frequency corresponds to approximately $1 \%$ of available surface sites becoming functionalized.

In order to determine how dependent these results are on nanotube diameter, we performed the same randomized functionalization calculations for a larger $(10,10)$ carbon nanotube (Fig. 9). Once again the same linear downward trend is observed, this time with best fits of $v=-0.996 x+$ $171.1 \mathrm{~cm}^{-1}$ and $v=-1.078 x+171.0 \mathrm{~cm}^{-1}$ for hydrogenation and fluorination, respectively. The gradients are almost identical to those obtained for the $(6,6)$ nanotube, which tentatively encourages us that the above-mentioned rule of thumb, i.e., a $1 \mathrm{~cm}^{-1}$ downshift in RBM frequency for every $1 \%$ of surface sites that are functionalized may be valid for a range of metallic single-walled nanotubes.

\section{CONCLUSIONS}

In this paper, we have used DFT calculations to investigate the effects of chemical functionalization on both the resonance energy and vibrational frequency of the RBM of metallic carbon nanotubes, using the $(6,6)$ and also $(10,10)$ nanotubes as examples. We see a general downward trend in the $M_{11}$ Van Hove spacing with both positive and negative charging, predicting a redshift in the resonance excitation energy with 
both donor and acceptor doping for metallic nanotubes. In the $(6,6)$ tube, for charging beyond $0.8 \mathrm{e} / 24 \mathrm{C}$ atoms (either positive or negative), the Fermi level moves into the first Van Hove singularity peak, blocking $M_{11}$ resonance consistent with the loss of RBM intensity seen experimentally with functionalization [17].

Breaking down the various contributions of chemical functionalization to RBM frequency, based on the best-fit gradients of RBM frequency vs tube modification, we can summarize as follows. Charge transfer effects seem potentially more important in modifying RBM frequency in metallic nanotubes than changes in the effective mass of the nanotube carbon atoms. Of particular importance, while negative charging of the nanotube causes slight downshifting of the RBM, positive charging of the nanotube, for example, charge transfer to halogens, causes much larger downshifts. By eliminating other factors, we have demonstrated that covalent bonding and subsequent change in the force constants of $\mathrm{C}-\mathrm{C}$ bonds in the tube is a most significant factor in RBM frequency shifts.

The spatial distribution of the functional groups, as long as reasonably homogenous, does not appear to significantly alter RBM frequency. For atomic functional groups, such as hydrogen and fluorine, there is a high degree of similarity in their behavior. The RBM frequency in these metallic tubes is linearly proportional to the degree of functionalization, with a $\sim 1 \mathrm{~cm}^{-1}$ downshift in RBM frequency for each $1 \%$ of carbon surface sites functionalized. The linear proportionality may open avenues for quantitative analysis of relative degree of functionalization, for example, by spatially mapping RBM frequency across a sample.
Larger functionalization groups cause an upshift as seen in experiment because the rigid body model for coupled vibration between the functional group and carbon atom is not applicable; instead, the functional groups restrict the motion of the $\mathrm{C}$ atom. Thus, the sign of the RBM frequency shift can give a qualitative indicator of the type of functionalization behavior in the system. A logical extension of this frequency shift behavior dependence on functional group size is that by varying chain length in a functional group, it should be possible to invert the RBM frequency shift direction with degree of functionalization from a downshift for short chains lengths to an upshift for longer chains.

Where comparison is possible, our results are in good qualitative agreement with experiment. It is clear that the next step with this paper is to extend it to also consider semiconducting nanotubes, which will be the focus of a later study. It would also be interesting to extend the study to include phonon self-energy to go beyond the static limit $[41,42]$.

\section{ACKNOWLEDGMENTS}

All French partners acknowledge Project No. ANR-2010BLAN-0819-04 "SPRINT" for funding. M.J.R. thanks the Swedish Research Council (Reg. No. 2012-3174) for financial support. Most computations were performed on resources provided by the Swedish National Infrastructure for Computing (SNIC) at PDC and HPC2N. This article is dedicated to Prof. Bob Jones on the occasion of his 70th birthday.
[1] S. Iijima, Nature 354, 56 (1991).

[2] S. Iijima and T. Ichihashi, Nature 363, 603 (1993).

[3] D. S. Bethune, C. H. Kiang, M. S. Devries, G. Gorman, R. Savoy, J. Vazquez, and R. Beyers, Nature 363, 605 (1993).

[4] S. Banerjee, T. Hemraj-Benny, and S. S. Wong, Adv. Mater. 17, 17 (2005).

[5] M. A. Herrero and M. Prato, Mol. Cryst. Liq. Cryst. 483, 21 (2008).

[6] A. Di Crescenzo, V. Ettorre, and A. Fontana, Beilstein Journal of Nanotechnology 5, 1675 (2014).

[7] H. Kuzmany, A. Kukovecz, F. Simon, A. Holzweber, C. Kramberger, and T. Pichler, Synth. Met. 141, 113 (2004).

[8] A. M. Rao, E. Richter, S. Bandow, B. Chase, P. C. Eklund, K. A. Williams, S. Fang, K. R. Subbaswamy, M. Menon, A. Thess, R. E. Smalley, G. Dresselhaus, and M. S. Dresselhaus, Science 275, 187 (1997).

[9] F. Tuinstra and J. L. Koenig, J. Chem. Phys. 53, 1126 (1970).

[10] C. Thomsen and S. Reich, Phys. Rev. Lett. 85, 5214 (2000).

[11] J. Kurti, G. Kresse, and H. Kuzmany, Phys. Rev. B 58, R8869 (1998).

[12] A. M. Rao, J. Chen, E. Richter, U. Schlecht, P. C. Eklund, R. C. Haddon, U. D. Venkateswaran, Y. K. Kwon, and D. Tomanek, Phys. Rev. Lett. 86, 3895 (2001).

[13] S. M. Bachilo, M. S. Strano, C. Kittrell, R. H. Hauge, R. E. Smalley, and R. B. Weisman, Science 298, 2361 (2002).

[14] S. S. Islam and K. A. Shah, Ieice Electronics Express 3, 5 (2006).
[15] C. Bergeret, J. Cousseau, V. Fernandez, J. Y. Mevellec, and S. Lefrant, J. Phys. Chem. C 112, 16411 (2008).

[16] H. Kataura, Y. Kumazawa, Y. Maniwa, I. Umezu, S. Suzuki, Y. Ohtsuka, and Y. Achiba, Synth. Met. 103, 2555 (1999).

[17] J. Y. Mevellec, C. Bergeret, J. Cousseau, J. P. Buisson, C. P. Ewels, and S. Lefrant, J. Am. Chem. Soc. 133, 16938 (2011).

[18] C. Menard-Moyon, N. Izard, E. Doris, and C. Mioskowski, J. Am. Chem. Soc. 128, 6552 (2006).

[19] A. Kukovecz, C. Kramberger, M. Holzinger, H. Kuzmany, J. Schalko, M. Mannsberger, and A. Hirsch, J. Phys. Chem. B 106, 6374 (2002).

[20] J. L. Bahr, J. P. Yang, D. V. Kosynkin, M. J. Bronikowski, R. E. Smalley, and J. M. Tour, J. Am. Chem. Soc. 123, 6536 (2001).

[21] S. Cambre, B. Schoeters, S. Luyckx, E. Goovaerts, and W. Wenseleers, Phys. Rev. Lett. 104, 207401 (2010).

[22] S. Kawasaki, K. Komatsu, F. Okino, H. Touhara, and H. Kataura, Phys. Chem. Chem. Phys. 6, 1769 (2004).

[23] P. E. Pehrsson, W. Zhao, J. W. Baldwin, C. H. Song, J. Liu, S. Kooi, and B. Zheng, J. Phys. Chem. B 107, 5690 (2003).

[24] Z. X. Guo, J. W. Ding, Y. Xiao, and D. Y. Xing, Nanotechnology 18, 465706 (2007).

[25] M. Muller, J. Maultzsch, D. Wunderlich, A. Hirsch, and C. Thomsen, Phys. Status. Solidi. B 244, 4056 (2007).

[26] J. P. Perdew and Y. Wang, Phys. Rev. B 45, 13244 (1992).

[27] R. Jones and P. R. Briddon, Semiconductors and Semimetals 51, 287 (1998). 
[28] M. J. Rayson and P. R. Briddon, Comput. Phys. Commun. 178, 128 (2008).

[29] M. J. Rayson and P. R. Briddon, Phys. Rev. B 80, 205104 (2009).

[30] P. R. Briddon and M. J. Rayson, Phys. Status. Solidi. B 248, 1309 (2011).

[31] C. Hartwigsen, S. Goedecker, and J. Hutter, Phys. Rev. B 58, 3641 (1998).

[32] J. P. Goss, M. J. Shaw, and P. R. Briddon, Theory of Defects in Semiconductors, Topics in Applied Physics, Vol. 104 (Springer, Berlin, 2007), pp. 69-94.

[33] A. H. Nevidomskyy, G. Csanyi, and M. C. Payne, Phys. Rev. Lett. 91, 105502 (2003).

[34] J. Kurti, V. Zolyomi, M. Kertesz, and G. Y. Sun, New J. Phys. 5 (2003).

[35] G. Y. Sun, J. Kurti, M. Kertesz, and R. H. Baughman, J. Phys. Chem. B 107, 6924 (2003).
[36] N. Wang and L. H. Guan, Nanoscale. 2, 893 (2010).

[37] M. Cardona and G. Guntherodt, Top. Appl. Phys. 50, 1 (1982).

[38] S. D. Costa, C. Fantini, A. Righi, A. Bachmatiuk, M. H. Rummeli, R. Saito, and M. A. Pimenta, Carbon 49, 4719 (2011).

[39] E. Dobardzic, J. Maultzsch, I. Milosevic, C. Thomsen, and M. Damnjanovic, Physica Status Solidi B-Basic Research 237, R7 (2003).

[40] R. Jones, J. Goss, C. Ewels, and S. Oberg, Phys. Rev. B 50, 8378 (1994).

[41] H. Farhat, K. Sasaki, M. Kalbac, M. Hofmann, R. Saito, M. S. Dresselhaus, and J. Kong, Phys. Rev. Lett. 102, 126804 (2009).

[42] K. I. Sasaki, R. Saito, G. Dresselhaus, M. S. Dresselhaus, H. Farhat, and J. Kong, Phys. Rev. B 78, 235405 (2008). 